\title{
Gérer les connaissances d'employés de service dans de grandes organisations hiérarchiques : le rôle fondamental des acteurs transversaux
}

\author{
Sarah NOGUES \\ Télé-Université du Québec, Canada \\ sanogues@teluq.ca \\ Diane-Gabrielle TREMBLAY \\ Télé-Université du Québec, Canada \\ dgtrembl@teluq.ca
}

\section{RÉSUMÉ}

Dans une économie de la connaissance, toute entreprise a intérêt à gérer les connaissances de ses employés à en créer de nouvelles dans un processus d'innovation continue. Reconnaître la logique communautaire des employés représente un défi pour les organisations à forte structure hiérarchique ayant de la difficulté à contrôler le travail des employés, notamment lorsque leur travail implique une interaction directe avec la clientèle. Notre article questionne la possibilité d'une écologie de la connaissance dans ces contextes organisationnels à l'aide des travaux théoriques d'Etienne Wenger, en prenant le cas du personnel navigant commercial dans les compagnies aériennes. Nous avons mené une recherche de terrain qualitative avec entretiens semidirigés auprès de 59 agents de bord et directeurs de vol provenant de trois compagnies aériennes occidentales. Nos résultats mettent en évidence le rôle central des acteurs transversaux pour la gestion des connaissances des communautés professionnelles dans les grandes entreprises à forte structure hiérarchique.

MOTS-CLÉS : Communautés de pratique, Gestion des connaissances, Innovation, Personnel Navigant Commercial, Service à la clientèle, Écologie de la connaissance

CODES JEL : O30, M5, R49 


\section{ABSTRACT \\ Managing the Knowledge of Frontline Employees in Hierarchical Organizations: The Fundamental Role of Transversal Actors}

In the knowledge economy, it is in every company's interest to engage in managing and creating knowledge in a process of continuous innovation. For large, highly hierarchized companies who seek to control their employees' work, recognizing the latter's communautarian logic and implicit practices is challenging, especially when a customer service is involved. Our paper questions the possibility of a knowledge ecology in this type of organizations based on the work of Etienne Wenger. We study the case of commercial airline cabin crews. We led a qualitative field research with semi-directive interviews with 59 flight attendants and service directors from three Western

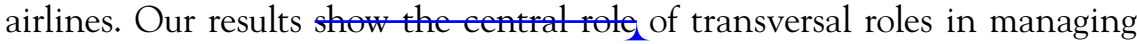
the knowledge of professional communities in large companies with a strong hierarchical structure.

KEYWORDS: Community of Practice, Knowledge Management, Innovation, Cabin Crews, Customer Service, Knowledge Ecology

JEL CODES: O30, M5, R49

À l'ère de l'économie du savoir, «toutes les entreprises sont par essence des organisations de la connaissance » (Brown, Duguid, 1998, p. 91, traduction libre) et doivent créer de nouvelles connaissances pour innover de manière continue afin de conserver leur avantage dans l'industrie (Nonaka, Takeuchi, 1995).

Pour ce faire, il leur faut capitaliser sur les connaissances créées par leurs employés. En effet, ceux-ci développent des connaissances « situées » et ancrées dans le contexte de leur pratique, potentiellement vectrices d'innovation (Brown, Duguid, 1991 ; Lave, Wenger, 1991 ; Orr, 1990 ; Wenger, 1998). Selon une vision constructiviste de la connaissance, celle-ci est produite collectivement dans le cadre de «communautés de pratique » où les acteurs se rencontrent et échangent pour trouver de nouvelles solutions à des problèmes communs (Lave, Wenger, 1991 ; Wenger, 1998, 2000, 2002). Ces « espaces sociaux d'apprentissage " (Wenger, 2009) font souvent partie de systèmes plus globaux, tels qu'une organisation formant une " constellation » ou un «paysage » de pratiques (Chanal, 2000 ; Wenger, 1998 ; Wenger, 2010). Lorsqu'il y a convergence entre la logique des communautés et la logique institutionnelle des organisations desquelles elles dépendent, et lorsque les communautés inter-reliées communiquent et s'enrichissent mutuellement, on voit se dessiner une véritable « écologie » de la connaissance (Brown, Duguid, 
1998 ; Chatti, 2012 ; Nonaka, Konno, 1998 ; Siemens, 2006). Tout ce système social d'apprentissage repose sur différents rôles et acteurs-clés, "artistes sociaux» (Wenger, 2009) dont les comportements de " citoyenneté d'apprentissage » permettent le fonctionnement (Star, Griesemer, 1989 ; Wenger, 2000, 2009, 2010).

Cependant, les communautés sont difficiles à envisager dans le contexte d'organisations hiérarchiques traditionnelles, préférant évoluer au sein de structures organiques (Roberts, 2006 ; Wenger, 2010). Par ailleurs, le concept de communauté de pratique a été critiqué autant par les praticiens que les chercheurs, les uns observant de nombreuses difficultés à mettre en place des communautés « pilotées » par la direction (Cohendet et al., 2010 ; HabhabRave, 2010 ; Harvey et al., 2013), les autres lui reprochant, entre autres, d'omettre la question du pouvoir dans ces systèmes sociaux d'apprentissage (Gherardi, 2009 ; Roberts, 2006). Si ce concept a permis de sensibiliser les organisations à l'importance des savoirs informels et émergents produits par la pratique des employés dans le processus d'innovation (Wenger, 2010), la gestion des connaissances situées dans des configurations organisationnelles hiérarchiques traditionnelles demeure problématique et méconnue (Aljuwaiber, 2016 ; Roberts, 2006).

Notre article s'intéresse à la gestion des connaissances situées des employés à l'interface avec la clientèle dans les organisations à structure hiérarchique verticale ou pyramidale. Nous prenons le cas du personnel navigant commercial (PNC), autrement dit des agents de bord ou "hôtesses de l'air/steward", dans les compagnies aériennes occidentales, soumises à une concurrence intensive (Bamber et al., 2009 ; Belobaba et al., 2016). Ce collectif professionnel présente une forte identité et travaille en équipages éphémères à des milliers de kilomètres de leur base. En même temps, le travail du PNC est fortement encadré par des procédures et standards sur le plan de la sécurité comme du service commercial. Notre analyse, qui repose sur des entretiens semi-dirigés auprès de PNC basés au Canada et en Allemagne et provenant de quatre compagnies aériennes différentes, a notamment permis de renseigner le rôle important transversaux situés aux frontières des communautés dans ce type de configurations organisationnelles, pour la gestion des connaissances et le développement des capacités d'apprentissage au sein de ces systèmes sociaux (Wenger, 2009, 2010). 


\section{Revue des écrits}

\section{Gestion des connaissances et innovation dans le secteur des services}

À l'ère de l'économie du savoir, les organisations reposent moins sur la force de travail de leurs employés que sur leur intelligence et leur degré qui li lification (Tremblay, 2015). Dans un contexte de concurrence accrue à ecnelle internationale, il devient particulièrement coûteux de " réinventer la roue » ou de refaire les mêmes erreurs (Becker, 2007) et l'apprentissage continu des travailleurs est un enjeu. Non seulement faut-il absorber les connaissances existantes dans un domaine donné, mais il est également nécessaire d'en développer de nouvelles pour obtenir ou conserver un avantage dans son industrie (Nonaka, Takeuchi, 1995).

De plus en plus, les atouts organisationnels deviennent intangibles (Davel, Tremblay, 2011 ; Saint-Onge, Wallace, 2003), ce qui en complexifie la gestion. Il faut trouver des façons d'exploiter au mieux les connaissances produites, d'où l'avènement de la vaste discipline de la gestion des connaissances. Des exemples japonais ont montré que la création de nouvelles connaissances organisationnelles et leur gestion constituent la clé de l'innovation (Nonaka, Takeuchi, 1995). Ainsi, la gestion des connaissances peut être entendue comme "la pratique de capturer et de développer les connaissances individuelles et collectives au sein d'une organisation, dans le but de les utiliser pour la promotion de l'innovation à travers le transfert de connaissances et l'apprentissage continu »(Hallin, Marnburg, 2007, p. 3, traduction libre). La gestion des connaissances et l'innovation sont l'affaire de toute entreprise et de tous en son sein, du personnel de production ou de service jusqu'à la direction (Brown, Duguid, 1998 ; Nonaka, Takeuchi, 1995). Par ailleurs, l'innovation n'est pas seulement technologique, mais souvent organisationnelle ou sociale (Tremblay, 2014). Elle repose sur la prise en compte et la gestion des connaissances individuelles et collectives des employés, notamment en matière de contact avec la clientèle dans le secteur des services (Hipp, Grupp, 2005 ; Kahle, 2002 ; Hallin, Marnburg, 2007).

Dans la gestion des connaissances, on s'intéresse connaissances «tacites » développées par les employés, soit des connaissances intuitives apprises avec l'expérience (Polanyi, 1958). En effet, ces connaissances qui résident « dans la tête » des praticiens sont reconnues pour être sources d'avantages compétitifs, car difficilement reproductibles par les compétiteurs (Barney, 1991 ; Nonaka, Takeuchi, 1995). On les distingue des connaissances explicites, qui sont aisément transminsibles et codifiables. À partir de cette 
distinction, les chercheurs Nonaka et Takeuchi (1995) ont distingué quatre modes de création de connaissances. Le premier mode est celui de la socialisation, de " tacite à tacite ", où les connaissances tacites sont partagées via la pratique, l'imitation et l'observation. Le deuxième mode est celui de l'externalisation, de «tacite à explicite », où les connaissances tacites sont articulées en concepts explicites, notamment par le biais de métaphores. En troisième lieu, la combinaison est un processus d'« explicite à explicite », où l'on intègre différents ensembles de connaissances dans un système. Enfin, l'internalisation est un processus $\mathrm{d}^{\prime}$ ' explicite à tacite », où l'on intègre des connaissances explicites dans ses bases de connaissances tacites sous forme de savoir-faire (Nonaka, Takeuchi, 1995).

\section{Communautés de pratiques et connaissances situées}

Les travaux sur l'innovation et la gestion des connaissances reconnaissent généralement que celles-ci émergent de la pratique des acteurs et sont produites collectivement, faisant l'objet d'un « savoir en action » ou de « connaissances situées » (Amin, Roberts, 2008). L'origine de cette vision se rapporte aux travaux ethnographiques de Lave et Wenger (1991) étudiant les dynamiques d'apprentissage au sein de " communautés de pratique » $(\mathrm{CoP})$, soit des « système[s] de relations entre des personnes, des activités et le monde » (Lave, Wenger, 1991, p. 98). Les chercheurs prônent une vision constructiviste de l'apprentissage où les connaissances sont produites à travers la pratique collective. Les membres d'une CoP sont typiquement liés par une compréhension commune et développée collectivement du sens de leur communauté, ils présentent des relations soutenues dans le cadre d'un engagement mutuel dans la pratique et partagent un répertoire de ressources communes, tels qu'un jargon, des outils, des histoires, etc. (Wenger, 1998, 2000). L'identité est un aspect fondamental des CoP (Eckert, 1989 ; Wenger, 1998).

Ce concept a suscité de nombreux travaux dans la littérature en systèmes d'information et en gestion, dépassant sa discipline d'origine sur l'apprentissage. Ces développements ont notamment donné lieu à un passage de la notion de communautés «spontanées ", émergeant de manière informelle entre praticiens, à la notion de communautés " pilotées » mises en place par les directions (Cohendet et al., 2010) comme instrument de gestion des connaissances dans la perspective d'innovation (Habhab-Rave, 2010). Dans ces communautés, le leadership, la culture organisationnelle, le sentiment d'appartenance ainsi que la motivation individuelle à participer au sein des communautés constituent d'importants facteurs de succès (Retna, Ng, 2011 ; Tremblay, 2005). Par ailleurs, des concepts connexes ont été développés, amenant à découvrir de nouveaux systèmes sociaux d'apprentissage par la pratique 
(Amin, Roberts, 2008 ; Aljuwaiber, 2016 ; Linkvist, 2005). Amin et Roberts (2008) décrivent par exemple des "savoirs professionnels ", où des praticiens d'une profession donnée mêlent un apprentissage basé sur des connaissances explicites apprises lors de formations avec des connaissances tacites apprises sur le terrain par socialisation. Ces communautés professionnelles présentent des connaissances d'experts spécialisés, acquises au cours de longues périodes de formation et d'éducation. Elles existent sur une longue durée et changent lentement, selon une innovation fortement liée par les standards de la profession. Par ailleurs, une fois que les membres de ces communautés professionnelles ont appris et acquis une certaine maîtrise du métier par socialisation avec les autres praticiens, une communication virtuelle semble suffire au partage et développement de nouvelles connaissances (Amin, Roberts, 2008).

Cependant, on peut se demander dans quelle mesure les connaissances tacites développées collectivement sur le terrain s'arriment avec les objectifs organisationnels et attentes de la direction. Bien que les communautés professionnelles soient dépendantes des standards de la profession (Amin, Roberts, 2008), peu importe le degré auquel leurs membres sont soumis à des directives dans le cadre de leur travail, les travailleurs «produisent une pratique qui reflète leur propre engagement avec la situation» (Wenger, 2010, p. 2, traduction libre). Dès lors, les employés qui apprennent et travaillent ensemble sur le terrain développent leur propre interprétation du travail et ne peuvent être vus comme de simples exécutants (Bechky, 2003 ; Brown, Duguid, 1991 ; Orr, 1990 ; Wenger, 1998). Il tend ainsi à exister une dichotomie entre les règles de travail « canoniques » telles que conçues au niveau du management et les pratiques quotidiennes des acteurs qui effectuent le travail (Brown, Duguid, 1991 ; Orr, 1990). Dans ce sens, il a été mentionné que les communautés sont plus susceptibles de s'épanouir dans des organisations à structure organique avec des employés qualifiés et autonomes, par opposition aux organisations à structure hiérarchique traditionnelle et pyramidale (Roberts, 2006 ; Wenger, 2010). Du point de vue du management, l'empowerment des employés de la base (service ou production) dans ce type d'entreprises ne va pas de soi (Bowen, Lawler, 1992). Dès lors, la gestion des connaissances situées dans une perspective d'innovation au sein de ces configurations organisationnelles traditionnelles pose question. 


\section{Cadre conceptuel}

\section{Entre imputabilité verticale et horizontale}

La question d'une disjonction entre la logique de la direction et celle des travailleurs a été abordée dans les travaux de Wenger (2009, 2010). Le chercheur reconnaît l'existence de deux axes de pouvoir au sein des organisations. D'une part, les employés sont liés par une dynamique d'imputabilité horizontale au sein de leur communauté de praticiens, à travers l'engagement dans l'activité conjointe, la négociation du sens donné à la pratique et l'identité commune, selon une logique de reconnaissance par les pairs. D'autre part, ils sont imbriqués dans une structure d'imputabilité verticale, soit la logique institutionnelle et hiérarchique de l'organisation qui les emploie, avec son autorité décisionnelle, sa logique de gestion des ressources, ses politiques et réglementations, etc. Un problème récurrent, souligne Wenger (2010), est l'invisibilité de l'axe d'imputabilité horizontale aux yeux du management, bien que ces règles et codes informels soient tout aussi liants et engageants que les règles découlant du système hiérarchique.

\section{Rôles transversaux}

Face à ce problème, Wenger (2010) met en avant l'importance de divers rôles situés aux frontières des communautés, notamment entre ces deux axes de pouvoir. Il parle notamment d'« artistes sociaux» qui inspirent des comportements de « citoyenneté d'apprentissage » au sein des communautés (Wenger, 2009). Traditionnellement, la littérature sur les CoP reconnaît un certain nombre de rôles, comme entre autres les rôles de traduction et d'interface. Les premiers traduisent les intérêts d'une communauté donnée du point de vue d'une autre ; les seconds participent à la pratique de plusieurs communautés, permettant une circulation des connaissances (Star, Griesemer, 1989). Ces acteurs-interface sont dans une position délicate, courant d'une part le risque d'être trop impliqués dans une communauté pour être perçus comme légitimes par l'autre, ou alors courant carrément le risque d'être exclus (Chanal, 2000 ; Wenger, 1998). Par rapport à cette typologie classique, Wenger (2009, 2010) met en avant un nouveau type d'acteurs, les acteurs « transversaux ", fournissant des connexions dans un contexte où les structures d'imputabilité horizontale et verticale sont disjointes. Selon le chercheur, on comprend que les « artistes sociaux » sont souvent des acteurs liminaux qui se situent aux frontières des communautés où l'identité est incertaine, des lieux autant source de conflit que d'innovation. Or, le rôle de ces artistes sociaux, en 
particulier celui des acteurs transversaux, est méconnu. Ce dernier à notre connaissance n'a jamais été étudié empiriquement.

\section{Une écologie de la connaissance}

Pour Wenger (2010), la CoP est un système social « simple ». Un système social « complexe » est quant à lui composé de communautés inter-reliées, justement grâce aux acteurs et processus transversaux ou frontaliers. On peut rapprocher ce concept de système social complexe à de celui d'écologie ou écosystème de la connaissance (Brown, 1999, 2002 ; Brown, Duguid, 1998 ; Pata, 2009 ; Siemens, 2006). L'écologie est un environnement de partage de la connaissance (Siemens, 2006) et peut être définie comme « un paysage complexe et à haute intensité en connaissances " qui émerge de connexions entre travailleurs du savoir (Chatti, 2012, p. 837, traduction libre). Selon Siemens (2006), la santé de chaque réseau personnel d'apprentissage, autrement dit des capacités d'apprentissage de chaque individu, est étroitement liée à l'état global de l'écologie dans laquelle se trouve l'apprenti. Une écologie de la connaissance est typiquement ouverte, émergente, auto-organisée, complexe, adaptative et décentralisée (Chatti, 2012). De ce point de vue, instaurer une écologie de la connaissance dans le contexte d'une organisation hiérarchisée avec processus descendants (top-down) paraît contre-intuitif.

Wenger (2010, 2009) souligne toutefois que dans les systèmes sociaux complexes, un axe de pouvoir vertical apparaît nécessaire. Dans bon nombre d'organisations, les axes verticaux et horizontaux fonctionnent de manière séparée. Or, pour encourager l'apprentissage continu à un niveau systémique, il faut les mettre en interaction, même s'ils demeurent inévitablement en tension. Lun des principaux défis pour les organisations concernées par l'innovation et l'apprentissage aujourd'hui est donc de développer des processus et des rôles transversaux permettant une «interface productive » entre axes verticaux et horizontaux au sein de systèmes sociaux complexes tels que de grandes organisations (Wenger, 2009, p. 17), contribuant ainsi à une écologie de la connaissance.

\section{Question de recherche}

Peu d'études s'intéressent aux organisations hiérarchiques et aux employés du secteur des services dans les écrits sur la gestion des connaissances et les CoP. Or, toute entreprise a besoin d'innover et de gérer les connaissances de ses employés (Brown, Duguid, 1998 ; Nonaka, Takeuchi, 1995), particulièrement là où ceux-ci doivent satisfaire les attentes de clients (Hallin, Marnburg, 2007 ; Khale, 2002 ; St-Onge, Wallace, 2003). Cependant, les compagnies à 
forte structure hiérarchique ont été désignées comme peu propices au développement de communautés de pratique (Roberts, 2006). Dès lors, dans quelle mesure la gestion des connaissances situées est-elle possible dans ces configurations ? Une écologie de la connaissance émergente, ouverte et adaptative, est-elle envisageable ? Nous nous demandons notamment comment se manifestent les interactions entre les axes d'imputabilité vertical et horizontal (Wenger, 2009, 2010) entre le management et des employés de la base, en tâchant d'identifier les acteurs et processus transversaux et liminaux. En effet, Wenger (2010, p. 14) nous informe de l'existence d'un « acteur fournissant des connexions transversales " entre ces deux axes, toutefois nous n'en savons pas plus sur la manière dont il le fait, ou quelles sont les caractéristiques de cet acteur. Afin de répondre à ces interrogations, nous prenons le cas du PNC, personnel de terrain en contact prolongé avec la clientèle dans le milieu compétitif de l'aviation commerciale (Belobaba et al., 2016). En nous basant sur l'expérience des praticiens, nous pourrons répondre aux appels à contributions pour une analyse de contextes institutionnels et organisationnels encore inexplorés dans la littérature sur la gestion des connaissances (Aljuwaiber, 2016 ; Retna, Ng, 2011), une analyse des relations de pouvoir et interactions entre les communautés et le management des organisations (Roberts, 2006), ainsi que des éléments d'analyse supplémentaires sur la dynamique de groupe au sein des communautés (Habhab-Rave, 2010).

\section{Méthode}

Le présent article s'inscrit dans un projet de recherche plus global sur le travail du PNC, dans le cadre d'un partenariat avec les divisions syndicales de PNC au Canada et en Europe. Le projet comportait un volet quantitatif avec distribution d'un questionnaire à grande échelle et un volet qualitatif avec entrevues semi dirigées, dans le cadre duquel nous avons rencontré en tout 59 agents de bord et directeurs de vol canadiens et allemands. Le présent article se base sur les résultats du volet qualitatif de l'étude seulement. En effet, étant donné le caractère exploratoire de notre objet d'étude au sujet d'acteurs liminaux et de ce type de configuration organisationnelle dans la littérature sur $\mathrm{GC}$ et $\mathrm{CoP}$, nous avons mené une recherche exploratoire, qualitative avec entretiens semi-dirigés.

Nous avons effectué une revue des écrits dans des bases de données en gestion et sciences sociales ainsi qu'au sein de revues spécialisées (Google scholar, Cairn.info, Business source complete, ABI/Inform, Journal of Air Transport Management, Tourism Management) à l'aide des mots-clés suivants : personnel navigant commercial/cabin crews; agents de bord/flight 
attendants ; travail/work ; pratiques/practice ; communauté de pratique/community of practice, Knowledge management and cabin crews ; knowledge ecology ; situated learning ; situated knowledge ; professional communities ; innovation. Avec l'accord des syndicats, les participants ont été contactés par courriel et invités à participer à une entrevue semi-dirigée. Une première série d'entrevues avec participants volontaires (qui avaient laissé leur courriel à la fin du questionnaire en ligne) s'est déroulée entre août et décembre 2016 auprès de PNC de deux compagnies aériennes canadiennes (Cie 1 et Cie 2), en personne et par téléphone, selon les disponibilités des participants. Une deuxième série d'entrevues s'est déroulée par téléphone et visioconférence entre mars et juin 2017 auprès de 18 PNC allemands, tous sauf un provenant d'une même grande compagnie du pays (Cie 3 et Cie 4). Les entrevues sont d'une durée moyenne de 75 minutes.

Les participants étaient tous membres du PNC, collectif de travail se rapprochant de la communauté professionnelle au sens d'Amin et Roberts (2008). En effet, le PNC est soudé autour d'une histoire commune de lutte pour la reconnaissance de leur statut de professionnel de la sécurité (Lessor, 1984 ; Whitelegg, 2007), partageant un jargon unique, des histoires et des rituels communs, notamment en escale (Dubey, Martin, 2001 ; Poirot-Delpech, 2016 ; Simpson, 2014). Ils sont également fortement liés par les normes de leur profession, tous passant par une formation initiale intensive de 5 à 8 semaines dépendamment des compagnies. En matière de sécurité comme de service commercial, le travail du PNC est prescrit et repose sur un ensemble de procédures standardisées apprises par cœur lors de cette formation initiale et qui sont réitérées par écrit dans les manuels ou tablettes numériques que les agents emportent à bord (connaissances explicites). Cependant, leur travail impliquerait un "savoir-faire intuitif » plus vaste : dans une étude auprès d'agents de bord français, Barnier (1999) remarque que ceux-ci « s'arrangent » et « pactisent » avec les règles établies du service commercial pour l'atteinte de l'objectif commun. Il existe ainsi un mélange entre savoirs explicites et intuitifs ou tacites. Dans ce cadre, on se demande quelle est la relation entre les connaissances tacites et la logique horizontale du PNC et la logique verticale du management qui reflète les standards de la profession.

Avant chaque entrevue, un formulaire de consentement a été présenté aux participants. Nous avons demandé à chacun s'il ou elle acceptait l'enregistrement audio, ce que personne n'a refusé. Une fois les fichiers audio recueillis et un sentiment de saturation des données atteint, autrement dit une impression de redondance (Glaser, Strauss, 1967), les entrevues en français et en anglais ont été retranscrites mot pour mot par deux assistants de recherche. Nous avons attribué des noms fictifs aux participants. Les données 
demeurent conservées sur une clef USB nécessitant un code d'accès. Elles ont ensuite été analysées selon la technique de l'analyse de contenu qualitative, en repérant les thèmes ou schémas communs émergeant du discours des participants (Drisko, Maschi, 2016 ; Glaser, Strauss, 1967). Nous nous plaçons ainsi dans une approche interprétative et constructiviste de la recherche, en ce sens que nous visons une "compréhension riche d'un phénomène, ancrée dans le point de vue et dans le sens que les acteurs sociaux donnent à leur réalité » (Savoie-Zacj, 1997, p. 337). Une liste de thèmes ou codes a été établie manuellement et retravaillée plusieurs fois au moyen d'allers-retours fréquents aux transcriptions, notes de terrain et comptes rendus (Patton, 1999).

\section{Résultats}

Nous avons recherché une certaine diversité de situations et avons rencontré 36 agents de bord et 23 directeurs de vol. Chaque vol comprend au moins une directrice ou un directeur de vol, chargé de veiller à ce que le service en cabine se déroule selon les normes et à ce que la cabine soit sécuritaire pour tous. $\#_{2}$ est donc tenu de voir à ce que les agents de bord effectuent correctement leur travail en matière de service commercial comme de sécurité. Lancienneté moyenne de nos participants est de 17 ans au Canada et 9,5 ans en Allemagne. Notre groupe comporte 40 femmes et 19 hommes. Les participants proviennent quatre compagnies aériennes : Cie 1, une ligne régulière canadienne ; Cie 2, une compagnie moyenne gamme canadienne ; Cie 3, une ligne régulière basée en Allemagne et Cie 4, une compagnie moyenne gamme Allemande. Cie 1 emploie environ 7000 PNC, Cie 2 environ 2000 et Cie 3 plus de 20 000. Tout d'abord, nous décrivons les caractéristiques des structures d'imputabilité horizontale et verticale, en mettant en évidence leurs points de jonction. Ensuite, nous analysons les tensions et disjonctions entre la communauté du PNC et le management.

\section{Structure verticale : la logique institutionnelle}

Nous rapportons ici les acteurs et lieux de transmission de connaissances entre le PNC et d'autres entités de la compagnie.

\section{Hiérarchie d'acteurs}

Pour le PNC, deux types de hiérarchies sont à distinguer : une « au sol », et une « en vol». Dans le premier cas de figure, les PNC sont redevables de plusieurs départements, notamment le service en vol qui veille au respect des normes en matière de service à la clientèle, le département de la sécurité, le 
service de planification des horaires, etc. Le « management » est une entité nébuleuse aux yeux de la plupart de nos participants, à raison d'un superviseur par base. En vol, le groupe du PNC est officiellement sous l'autorité des pilotes et du directeur de vol, ce dernier effectuant une coordination entre le cockpit et la cabine. Du point de vue de l'État, il est responsable de la sécurité en cabine, et du point de vue de la compagnie responsable de veiller à l'application des normes du service commercial.

\section{Briefing}

Avant chaque vol, la directrice ou le directeur de vol s'assure que l'équipage connaît les procédures de sécurité et est au courant des dernières nouveautés en matière de service, qui normalement ont été communiquées à tous par un mémo. Outre rappeler les procédures de sécurité, il « donne le ton » du vol et communique ses « attentes ». C'est ainsi qu'Olivier, directeur de vol chez Cie 2, " un gars du service à la clientèle ", va demander à chaque me son équipage de se rappeler une situation de leur vie personnelle dans laquelle ils auraient reçu un mauvais service, de manière à développer 'empathie chez de conté, Maya, directrice de vol chez Cie 3, leur demande de penser à " six manières différentes d'offrir le thé ou le café » afin que chaque passager se sente valorisé, différencié de son voisin. En même temps, le briefing constitue surtout un moment de transmission descendante de connaissances explicites provenant du management.

\section{Comités et formations}

L'implication de nos participants dans divers comités au sein de la compagnie était fréquente, si bien que les agents de bord et directeurs de vol y participant ramenaient à bord un nouveau bagage de connaissances, se disséminant ensuite d'équipage en équipage. Les agents avaient par exemple la possibilité de participer à des comités d'amélioration du service à la clientèle, de participer aux entretiens d'embauche ou de devenir formateurs. Ainsi, les institutions au sein des compagnies aériennes permettent la circulation descendante des connaissances, de par l'implication des PNC dans les comités de la hiérarchie au sol et l'internalisation de ces connaissances par la suite sur le terrain. Dans la logique verticale du management, le poste de directeur de vol constitue un rôle clé en matière de gestion des connaissances. Comme nous allons le voir, il est également important dans la logique communautaire du PNC. 


\section{Structure horizontale : les façons de faire du PNC}

Malgré un travail prescrit, bon nombre de participants se sentaient comme des " travailleurs autonomes » à bord : " on s'organise très bien entre nous ", nous assure Cassandre, agente de bord chez Cie 2. L'équipage en cabine est décrit comme un véritable " écosystème » selon Alex, directrice de vol chez Cie 2, non sans insister sur l'éloignement et la différence qui caractérisent la communauté soudée du PNC par rapport aux acteurs au sol.

\section{Un " écosystème " reposant sur le leadership du directeur de vol}

Plutôt que de respecter les postes assignés, on tend plutôt à s'organiser en fonction des forces et capacités de chacun : Jane, agente de bord chez Cie 1, se retrouve souvent à prendre la position de la galley ou cuisine, à cause de sa préoccupation pour l'efficacité du service et de sa nature « organisée ». Elle laisse à d'autres le soin de s'occuper du volet « relations publiques » en cabine d'ent peut-être plus de patience qu'elle auprès des passagers, mais sont moins « rapides » pour les besoins du service. Tania, de par sa formation en travail social, est souvent désignée pour aller apaiser des passagers traversant des crises personnelles. Si quelqu'un ne fait pas sa part, un mode de « pression par les pairs » se met en place pour rétablir l'harmonie au sein de l'équipage.

Malgré un travail d'apparence routinière, beaucoup de nos participants rapportaient qu'« aucun vol n'est pareil », et que l'on s'en va au travail dans « l'inconnu » à chaque nouveau vol. On est toujours sur le qui-vive et l'on s'attend à rencontrer des situations inédites. Des réflexes s'affûtent cependant, qui permettent, lorsque mis en commun et discutés avec les collègues, de créer de nouvelles connaissances situées dans la pratique du métier d'agent de bord. Dans l'exemple suivant, Catherine rapporte qu'avec davantage de collègues, elles seraient assez nombreuses pour se concerter et détecter ensemble un passager dont la consommation élevée de Coca-Cola peut laisser suspecter une d'alcool importante à partir d'alcool qu'il aurait lui-même apporté à bord :

«Si on était plus, on serait comme : 'Encore un eoke ? Mmhh...' (rires) Là tu jases avec un peu... juste pour voir... ». (Catherine, agente de bord, Cie 1 low cost)

Malgré cette organisation en complémentarité, les agents comptent sur la directrice ou le directeur de vol pour assurer une dynamique au sein de l'équipage. Par exemple, on s'y réfère lorsqu'une situation inhabituelle se présente et que l'on se trouve dans une zone grise. On attend tout de même de ce dernier un leadership participatif, soit de demander l'avis des agents et de 
se concerter pour trouver une solution aux situations données. Rien de pire qu'un directeur de vol qui est « sur votre dos », qui vous « micro-manage » et qui se prend pour un « superviseur » tout en se mettant à paniquer lorsque la situation dégénère et, surtout, qui ne vous fait pas confiance. Dans ce cas, son autorité de directeur de vol risque de ne pas être respectée par les agents. En bref, il est souvent dit que le directeur de vol « donne le ton » au vol et est respecté du point de vue de sa compétence et ses qualités de leader. Un grand nombre de participants avaient décidé d'accéder à ce poste après avoir été témoins du leadership inspirant de directeurs de vol en tant quagents de bord, ou au contraire pour éviter le mauvais leadership de certains qu'ils ne voulaient plus avoir à «subir », confiants de pouvoir « mieux faire ». Il y avait donc chez la plupart de nos participants directeurs de vol une volonté d'améliorer les pratiques à bord en accédant à ce poste.

\section{Socialisation et apprentissage}

Entre agents de bord, il existe un partage de pratiques dans le cadre d'un travail qui s'apprend essentiellement " sur le tas », hormis les procédures opérationnelles apprises par cœur en formation pour le volet sécurité. On remarque une ouverture à apprendre des plus jeunes : « tu peux m'en apprendre comme je peux t'en apprendre », rapporte Sylvie, agente de bord chez Cie 2. Chez Cie 3, Helga s'efforce de donner des astuces qui permettront aux plus jeunes collègues de gagner en «maturité », comme de ne pas rentrer dans le jeu de passagers qui essaient d'obtenir plus que ce qui leur est dû. Nous observons ainsi un échange de pratiques entre agents de bord séniors et juniors. La mixité des équipages en termes de génération était valorisée. Nous voyons en revanche que Catherine, employée dans la branche low cost de Cie 1 où la grande majorité de ses collègues a « moins de 25 ans » et où il n'y a pas de directeur de vol officiel, éprouve de la difficulté à initier un partage de pratiques. La tentative même de bénéficier de l'expérience de quelqu'un plutôt que de " réinventer la roue » lui vaut de se faire voir comme incompétente. Par ailleurs, plusieurs de nos participants, toutes compagnies confondues, ont trouvé chez les nouvelles recrues un "manque d'initiative », tous ne manifestant pas la volonté d'apprendre. Flavie, directrice de vol chez Cie 2, observe avec regret que les agents de bord «ne se responsabilisent pas ». Pour sa part, Cynthia trouve qu'il est plus difficile de collaborer et trouver de nouvelles idées avec les plus jeunes :

« Je trouve que notre génération, je généralise (...) on est plus travailleurs, on a plus d'initiatives, plus d'idées, plus de solidarité ». (Cynthia, agente de bord, Cie 2) 
Cependant, la directrice ou directeur de vol est là pour rehausser le niveau et inspirer une amélioration des pratiques. Olivier, qui se voit comme un « vieux de la vieille » et un « gars du service à la clientèle », s'efforce de transmettre à chaque vol les fondamentaux de cet « art » que chacun développe « à sa manière ». Ses efforts ont notamment valu à Cie 2 un article élogieux dans la presse, un passager journaliste se trouvant impressionné de ce qu'on puisse lui présenter « autant d'options » (glace, citron) pour lui servir « un simple verre d'eau ». Olivier excelle ainsi à compenser la perte en " hardware » (nourriture, services) par du « software » (qualité relationnelle), et tâche de transmettre ses connaissances implicites aux équipages, par le biais de métaphores et en sollicitant l'imagination des agents.

Le PNC est donc un « écosystème » où l'on fait connaissance rapidement, où l'on s'organise en complémentarité et où l'on échange des pratiques et façons de faire. Le directeur de vol y a un rôle clé car il « donne le ton » du vol. Il est notamment reconnu et estimé du point de vue de son attitude envers l'équipage et de son style de leadership, plutôt que par son statut. Son titre de directeur ne doit pas tromper : «c'est l'un des nôtres ». En même temps, le directeur de vol a de hauts standards professionnels et tâche de tirer la barre vers le haut, là où tous les agents de bord ne sont pas nécessairement engagés dans la pratique et l'apprentissage continu. Cependant, certains participants canadiens rapportaient avoir le sentiment que leur compagnie veuille faire de ces acteurs de véritables gestionnaires, avec un rôle de contrôle tension parmi d'autres.

\section{Rencontres et disjonctions}

Nous expliquons ici les principaux aspects entravant la communication et la transmission de connaissances entre la communauté du PNC et la partie managériale.

\section{Une déconnexion entre le prescrit et la pratique}

Tout d'abord, les exigences du travail pouvaient être perçues comme « irréalistes ». Au Canada notamment, les participants étaient préoccupés par une loi récente ayant autorisé la réduction de personnel à bord, augmentant la charge de travail sur certains porteurs. De plus en plus, les directeurs de vol doivent faire face à des injonctions contradictoires. Par exemple, face à une période de turbulence prolongée, Isabelle, directrice de vol chez Cie 2, hésite à « couper dans le service » de sorte à éviter de stresser son équipage outre mesure. Toutefois, ce faisant, elle se compromettrait vis-à-vis du management pour qui le respect des standards est primordial. Pour Veronica, directrice de 
vol chez Cie 1, il existe une déconnexion entre le PNC et le management, qui veut contrôler ce qui se passe à bord sans pour autant comprendre de quoi il en retourne dans les faits.

Des participants canadiens comme allemands rapportaient par ailleurs un manque de canaux de communication ascendants en matière de connaissances. Les agents de bord ne sont pas consultés pour leurs connaissances ou idées par rapport à une situation particulière. Arno, agent de bord chez Cie 3, s'étonne que sa compagnie ne consulte pas les agents de bord concernant leurs connaissances vis-à-vis des besoins des passagers : après tout, ce sont bien eux qui les côtoient pendant des heures au quotidien et connaissent en détail leurs préférences. Par ailleurs, bon nombre de nos participants percevaient que les commentaires inscrits dans les rapports de vol ou autres courriels envoyés n'étaient pas lus, voire étaient « jetés à la poubelle ». Klaus, directeur de vol chez Cie 4 en Allemagne, conclut que son influence se limite strictement aux confins de la cabine :

«... because you make a lot of suggestions, you comment on things and ....it doesn't have [any] effect (...) you make suggestions and it never happens, or you never get a reply for example (...) Or they do just the opposite, they decide just the opposite decision you thought of (...) I can only make a difference on the flight that same day ». (Klaus, directeur de vol, Cie 4)

On le voit, ces directeurs de vol qui étaient arrivés à ce poste avec l'ambition de partager leur expérience et connaissances avec la partie managériale se sont trouvés déçus, et leurs connaissances n’ont pas été valorisées.

\section{Tensions identitaires}

Les participants de Cie 1 rapportaient une préoccupation commune : l'arrivée de nouveaux gestionnaires à bord quiennentapparemment observer le travail sur certains vols. Dans une ambiance de méfiance généralisée entre le groupe $\mathrm{PNC}$ et du management au sein de cette compagnie, cette « intrusion » va être « mal reçue par notre groupe », nous confie David, directeur de vol. Ayman, agent de bord, redoute que cette mesure ne vienne semer la discorde entre les membres de l'équipage. Jay pense que ce rôle va être " très solitaire ", notamment en escale où cette personne ne sera pas invitée avec l'équipage. En outre, il explique que ce regard managérial sur les pratiques du directeur de vol risque de nuire à l'efficacité du travail. En effet, si l'on ne lésine jamais avec la sécurité, il y a des «façons » de gérer son équipage de manière à ce que le vol soit plus confortable pour tout le monde, tout en respectant le temps imparti. La venue de gestionnaires risque de perturber 
ces « manières de faire » développées au fil du temps. Bien que ce pourrait être une occasion de rapprocher le management de la réalité du PNC et ainsi œuvrer ensemble pour un meilleur partage de connaissances entre les deux parties, l'on doute qu'une attitude coopérative soit possible : les rumeurs dans cette compagnie vont bon an mal an concernant les licenciements pour des raisons abusives et l'animosité semble forte entre la partie managériale et le groupe des PNC.

Par ailleurs, certains participants rapportaient une pression accrue sur les directeurs de vol à devenir eux-mêmes des " managers ", en assumant un rôle davantage de contrôle. La peur et l'intrusion du management dans la communauté des agents de bord, et plus particulièrement des directeurs de vol, semble donc menacer les pratiques du PNC dans cette compagnie. Cette menace identitaire arrive également avec une pression accrue à vendre des produits à bord, un rôle que nos participants avaient beaucoup de réticence à endosser ; en effet, alors qu'ils distribuaient gratuitement des repas auparavant, aujourd'hui pratiquement toutes les consommations doivent être vendues. Chez Cie 2, les « concours de vente » que la compagnie enjoint les directeurs de vol à organiser parmi l'équipage sont rarement appliqués en pratique. D'une part, parce qu'on n'a pas le temps, d'autre part, parce que l'on ne se voit pas du tout comme des « vendeurs ». C'est d'ailleurs la raison principale pour laquelle Alfonso, aujourd'hui agent de bord chez Cie 3, a quitté son emploi dans une compagnie low cost européenne pour reprendre le processus intégral d'embauche et de formation chez Cie 3, réputée plus respectueuse du rôle des agents de bord. Malgré tout, selon Alissa, qui a également travaillé dans une low cost auparavant, ces injonctions à adopter des pratiques de deư arrivent même dans cette compagnie, puisque la concurrence est vive entre les low cost et les anciennes compagnies régulières.

Ces tensions identitaires ne vont pas sans diviser les directeurs de vol, à tout le moins parmi nos participants canadiens. Certains vont se ranger du côté du management en appliquant davantage les règles de manière stricte ; cependant la tendance semble être davantage à se cristalliser du côté de l'équipage, surtout chez les directeurs de vol d'expérience. On le voit chez Bruno, directeur de vol chez Cie 2, qui a dû faire un « réajustement » de ses priorités : «Avant je travaillais pour ma compagnie et maintenant ma priorité c'est mes collègues de travail. » De son côté Veronica, directrice de vol chez Cie 1, prend de plus en plus un rôle de "coach" avec son équipage pour que les agents se rappellent de leurs droits, notamment face à la compagnie. Notons aussi que certains participants constataient une érosion des liens communautaires entre PNC où, notamment en raison de l'augmentation de la charge de travail, chacun est de plus en plus « dans sa bulle ». Même en escale, Romy, 
agente de bord chez $\mathrm{Cie} 3$, regrette que les nouvelles recrues préfèrent rester à l'hôtel connectées sur les réseaux sociaux plutôt que de participer aux excursions collectives à destination. Face à ce phénomène, certaines directrices de vol, comme Flavie chez Cie 2 ou Maya chez Cie 3, s'efforçaient de renforcer les liens communautaires en organisant des activités socialisantes en escale, pour conserver le « charme » de ce métier qui tend à en perdre :

«I try to show how beautiful the world is (...) Let's do something extraordinary that everybody is talking about in twenty years. That is something that is very, very important, in this job ». (Maya, directrice de vol, Cie 3)

Ainsi, l'identité du PNC est fragile, d'autant que l'on ne se côtoie que sur une période limitée, celle de la rotation. Nos résultats indiquent que les liens au sein de la communauté et avec les autres parties de l'organisation sont maintenus grâce à un acteur central, ou plutôt transversal : le directeur de vol.

\section{Discussion}

Nous nous sommes demandé si une écologie de la connaissance (Brown 1999/2002 ; Brown, Duguid, 1998 ; Chatti, 2012 ; Nonaka, Konno, 1998 ; Pata, 2009 ; Siemens, 2006) au sein de grandes organisations hiérarchiques, notamment reposant sur la performance d'employés de service en contact avec la clientèle, était possible. En somme, il s'agissait de voir si ces milieux organisationnels ont le potentiel de voir émerger un paysage complexe et à haute intensité en connaissances, formé de connexions entre travailleurs du savoir (Chatti, 2012) et de communautés de pratiques inter-reliées (Wenger, 2010). Ceci paraît crucial à l'ère de l'économie de la connaissance où il est nécessaire de capitaliser sur les connaissances des employés et d'en créer de nouvelles (Nonaka, Takeuchi, 1995), pour constituer des organisations intelligentes capables d'innover dans un marché hyper-compétitif comme celui de l'aviation commerciale (Belobaba et al., 2016). Dans les paragraphes qui suivent, nous discutons des contributions théoriques et pratiques de notre recherche.

\section{Contributions théoriques}

Nous avons analysé les processus et relations de transversalité selon la vision de PNC provenant de quatre compagnies aériennes. Nous avons mis au jour le rôle original des directeurs de vol, acteurs transversaux entre la 
logique communautaire des PNC et la logique institutionnelle des compagnies (Wenger, 2009, 2010). Nous enrichissons la littérature sur la gestion des connaissances et les $\mathrm{CoP}$ en proposant une figure archétypale de l'acteur transversal pour le cas de communautés professionnelles nécessitant réconciliation entre axe communautaire horizontal et axe institutionnel vertical.

\section{Acteur à la frontière de micro-communautés}

Soudé par des liens communautaires (Dubey, Martin, 2001 ; Lessor, 1984 ; Poirot-Delpech, 2016 ; Wenger, 2000 ; Whitelegg, 2007) et par les normes de la profession (Amin et Roberts, 2008 ; Barnier, 1999 ; Gueudar-Delahaye, 2012), le PNC se réunit lors de rotations que l'on peut qualifier, comme d'autres l'ont fait dans un autre contexte, de « micro-communautés » (Gjelsvik, 2002) où connaissances tacites et explicites circulent et se renouvellent à travers la pratique des agents. Le partage de trucs et astuces entre agents de bord de différents niveaux d'ancienneté est au cœur de cette dynamique, sur un mode de socialisation (Nonaka, Takeuchi, 1995). On observe également une interaction entre connaissances explicites acquises lors de fréquentation de comités et formations au sol, et connaissances intuitives plus anciennes acquises au fil de l'expérience des agents. La rotation est un temps où l'on apprend à se connaître rapidement, où l'on se concerte pour ne pas « réinventer la roue » (Becker, 2007) et où l'on trouve ensemble des idées pour gérer des situations inédites (Wenger, 2002), dans la mesure où « chaque vol est différent ». Dans ce sens, les connaissances sont produites collectivement (Lave, Wenger, 1991) et dans la solidarité (Chanal, 2000 ; Wenger, 1998). Cependant, le partage prend fin à chaque fin de rotation et il faut chaque fois « réapprendre à se connaître »; il est possible que ceci limite le potentiel d'innovation, mais cela peut aussi assurer le transfert des idées vers de nouvelles équipes.

Toute communauté est délimitée par des frontières (Chanal, 2000 ; Wenger, 1998)__ Le PNC partage des conditions communes qui en même temps constituent des frontières tangibles et intangibles vis-à-vis des autres groupes professionnels. Luniforme porté est unique au PNC. Les tâches exécutées en cabine sont centrées sur le service aux autres, s'opposant par exemple aux tâches techniques des pilotes. Par ailleurs, comme il a déjà été souligné, ceux-ci travaillent dans l'espace masculin du cockpit opposé à l'espace féminin de la cabine (Simpson, 2014). Historiquement, le PNC, les «volants » (Gueudar-Delahaye, 2012) ont eu des relations conflictuelles avec le management (Bacharach et al., 1996), personnel « au sol ». Nos résultats montrent un clivage persistant entre ces deux parties, particulièrement dans les compagnies canadiennes. Ces frontières font des rotations de véritables « microcosmes » ou « écosystèmes », pour reprendre les termes de nos 
participants. Par exemple, la venue à bord de nouveaux gestionnaires chez Cie 1 est mal perçue et suscite la méfiance au sein du PNC, qui va tendre à exclure cette personne. Seul un acteur est inclus et accepté par le PNC, malgré sa position à l'interstice entre gestion et travail de plancher : la directrice ou le directeur de vol. C'est donc sur cet acteur, posté à la transversalité, lieu autant source d'innovation que de malentendus, que notre attention se porte (Wenger, 2010).

\section{Entre logiques verticale et horizontale}

La question du pouvoir est au centre de notre réflexion (Gherardi, 2009 ; Roberts, 2006 ; Wenger, 2010). Quelle logique règne à bord, à 10000 mètres d'altitude dans l'espace clos de la cabine? Il existe chez le PNC une tension entre d'une part, une volonté de bien faire, de prendre le temps avec les passagers et de socialiser avec les collègues, et d'autre part, une pression à tout faire plus vite, plus efficacement et ce sans rogner sur les services offerts à bord. Les intérêts des agents et ceux de la direction ne sont pas diamétralement opposés : tous ont intérêt à faire un bon travail, les uns pour conserver l'harmonie au sein de l'équipage et passer un vol « agréable », les autres pour offrir un service à la hauteur des exigences sécuritaires et commerciales, et conserver la clientèle. Cependant, des tensions peuvent survenir au niveau de la définition de ce que constitue un «bon » travail. En effet, le PNC a une forte identité professionnelle (Lessor, 1986 ; Whitelegg, 2007) et se trouve confronté au terrain chaque jour ; au contraire, il est plus difficile pour la compagnie de connaître la réalité du terrain, puisque tout se passe dans les airs. La volonté d'en savoir plus sur la réalité concrète du travail des agents constituait certainement chez Cie 1 un motif d'envoyer de nouveaux gestionnaires à bord. Cependant, ceci se fait sans considérer la logique horizontale et les fortes frontières de la communauté du PNC (Brown, Duguid, 1991 ; Chanal, 2000 ; Orr, 1990 ; Wenger, 1998 ; Wenger, 2010). En particulier, cela envoie un message de méfiance envers les directeurs de vol qui, d'après nos résultats pourtant, font de leur mieux au quotidien pour fournir des connexions transversales entre ces deux parties sinon disjointes (Wenger, 2010), se gardant en équilibre sur la frontière entre PNC, pilotes, management et bien d'autres acteurs encore.

En effet, la directrice ou directeur de vol maintient un lien important entre le PNC et la compagnie. Il prévient notamment des potentielles dérives communautaires comme la pensée de groupe et la dépendance au sentier (Mutch, 2003 ; Roberts, 2006) lors de moments-clés comme le briefing où les standards de la compagnie sont réitérés en même temps qu'il explicite ses connaissances tacites, de sorte à inspirer les agents à développer leur propre expérience du 
terrain. Il dispose ainsi de capacités de traduction (Star, Griesmer, 1989) de la logique verticale de l'organisation dans la logique communautaire horizontale du PNC (Wenger, 2010). D'un autre côté, il tâche de les protéger contre une charge de travail trop élevée, par exemple en prenant la décision de « couper » dans le service, malgré les injonctions de la compagnie, pour faire en sorte que tous puissent prendre le temps de se ressourcer. C'est ainsi qu'il développe des «façons » d'adapter les normes organisationnelles au terrain. Par ailleurs, il prévient la fragmentation des liens communautaires et protège l'identité du PNC, aspect fondamental des communautés (Eckert, 1989 ; Wenger, 2000). Il le fait par exemple en organisant des activités socialisantes en escale, afin de créer des « moments inoubliables », nourrissant ainsi le répertoire commun de la communauté (Wenger, 1998). En outre, à l'instar des communautés pilotées (Cohendet et al., 2010), le directeur de vol met en œuvre un leadership en « donnant le ton » au vol, ce qui constitue un facteur de succès des communautés formées lors des rotations (Retna, Ng, 2011). Il instaure et réitère lors de chaque briefing une sorte de micro-culture, selon sa propre interprétation du travail et des attentes de la compagnie. L'exemple de la branche low cost de Cie 1 où il n'y a pas de directeur de vol formé en tant que tel à bord - mais seulement l'agent de bord le plus sénior - montre que cela n'encourage pas un partage de pratiques, surtout lorsque l'on a affaire à un équipage de faible ancienneté. Enfin, l'exemple évoqué d'un gestionnaire externe ne connaissant pas le travail du PNC montre à quel point l'expertise de terrain et la confiance des agents sont deux aspects essentiels que l'on doit retrouver chez un acteur transversal. Pour être accepté, il doit venir de l'intérieur de la communauté, soit débuter comme agent de bord. En cela, l'acteur transversal partage des caractéristiques de l'acteur interface (Star, Griesemer, 1989) : il doit conserver la confiance de chaque partie en maintenant chaque jour un délicat équilibre entre leurs logiques respectives, selon son bon jugement.

\section{Créateurs de nouvelles connaissances}

Pour finir, nos résultats indiquent que le directeur de vol participe à la création de connaissances au sein de la compagnie, selon les quatre modes de création de connaissances identifiés et expliqués par Nonaka et Takeuchi (1995). Le tableau ci-dessous en synthétise les principales caractéristiques, en reprenant des citations tirées de notre recherche.

En résumé, l'acteur transversal est essentiel à la gestion des connaissances des employés de la base et contribue à l'innovation au sein des entreprises. Dans un contexte où les entreprises reposent de plus en plus sur les atouts intangibles et l'intelligence de leurs employés (Davel, Tremblay, 2011 ; 


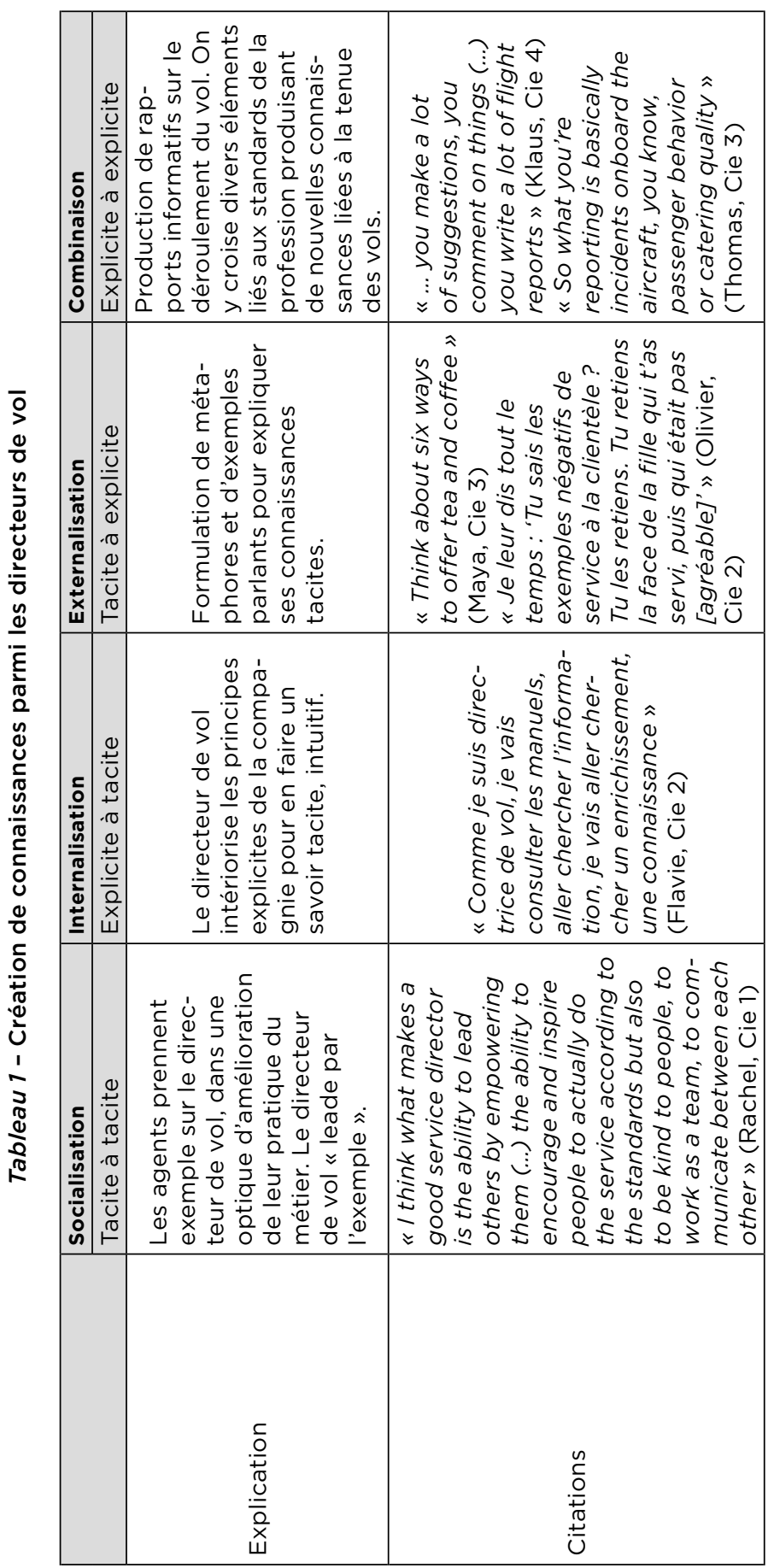


Saint-Onge, Wallace, 2003 ; Tremblay, 2015), l'acteur transversal contribue à la création de connaissances à travers des processus de socialisation, d'internalisation, d'externalisation et de combinaison (Nonaka, Konno, 1998 ; Nonaka, Takeuchi, 1995). Dans le secteur des services, il apparaît que l'acteur transversal favorise l'émergence d'une innovation sociale dans le service à la clientèle (Hipp, Grupp, 2005 ; Kahle, 2002 ; Hallin, Marnburg, 2007 ; Tremblay, 2014). Par son leadership participatif, il met en valeur les connaissances tacites que les employés développent sur le terrain (Brown, Duguid, 1991 ; Lave, Wenger, 1991 ; Orr, 1990 ; Polanyi, 1958) dans l'optique de trouver des solutions à des situations inédites (Wenger, 1998). Véritable « artiste social » (Wenger, 2009), il résout en partie la question du pouvoir dans ces configurations organisationnelles (Gherardi, 2009 ; Roberts, 2006) en constituant simultanément un vecteur des standards de la compagnie et un protecteur de la logique communautaire, notamment de l'identité et du répertoire commun (Eckert, 1989 ; Wenger, 1998) des employés de la base qu'il représente. Par ailleurs, il résout également les conflits liés au pouvoir présents au sein des équipages de par le statut officiel que lui confère son poste, certes, mais surtout de par son expertise et la confiance qu'il inspire aux agents. En effet, en l'absence de leader officiel et légitime selon les normes de la communauté, l'échange de pratiques dans une culture de partage et d'apprentissage n'est pas garanti. Si l'empowerment des employés de la base et les communautés de pratique ont été identifiés comme difficiles à mettre en place dans les configurations organisationnelles à forte structure hiérarchique (Bowen, Lawler, 1992 ; Roberts, 2006 ; Wenger, 2010), la présence d'acteurs transversaux rencontrant les caractéristiques énoncées semble créer un nid favorable à la formation de communautés au sein des équipes de travail, pouvant potentiellement constituer un instrument de gestion des connaissances dans la perspective d'innovation (Cohendet et al., 2010 ; Habhab-Rave, 2010). En réconciliant les logiques horizontales et verticales là où règnent traditionnellement une disjonction et des tensions liées au pouvoir (Chanal, 2000 ; Wenger, 1998 ; Wenger, 2010, Roberts, 2006), les acteurs transversaux ouvrent en théorie la voie à un "écosystème de la connaissance » (Brown, Duguid, 1998 ; Chatti, 2012 ; Nonaka, Konno, 1998 ; Siemens, 2006).

Cependant, notre recherche montre aussi qu'en pratique, à tout le moins pour le cas du PNC, la marge de manœuvre des acteurs transversaux en dehors de ce qui se passe sur le plancher ou plutôt dans les airs pour notre cas apparaît limitée. En effet, les comportements de citoyenneté d'apprentissage (Wenger, 2009, 2010) qu'ils mettent en œuvre ne trouvent pas de résonance pas au-delà de la cabine dans la mesure où l'on a le sentiment que les suggestions faites de manière ascendante ( bottom-up») ne se font pas entendre au niveau du management. Ainsi, le potentiel d'innovation porté par les communautés et 
les acteurs transversaux qui les représentent semble circonscrit au temps et au lieu de la rotation, empêchant une innovation sociale continue et systémique. Ainsi, si l'on a un système social d'apprentissage complexe fonctionnel sur le plan de la circulation et création des connaissances, a-t-on cependant affaire à une véritable écologie de la connaissance, vectrice d'innovation ?

\section{Implications pratiques}

Si l'on observe une circulation et création des connaissances au sein de la communauté professionnelle du PNC, avec infusion et internalisation de connaissances externes grâce aux diverses institutions (briefing, formation, comités), les compagnies aériennes semblent en revanche peu capitaliser sur les connaissances tacites des employés (Nonaka, Takeuchi, 1995).

\section{Investir dans des canaux de communication ascendants}

Dans le cadre d'une interaction entre employés et clients, il est important de prendre en compte les connaissances tacites des agents sur le terrain (Hallin, Marnburg, 2007 ; Hipp, Grupp, 2005 ; Kahle, 2002). Or, nos résultats indiquent un manque de canaux de communications ascendants. Mis à part une participante chez Cie 2 ayant rapporté s'impliquer dans un comité d'amélioration du service, il nous semble que le partage des connaissances n'est pas une priorité, voire est dissuadé par l'impression que les rapports écrits par les agents sont " jetés à la poubelle ». Les compagnies gagneraient à renforcer les incitations à partager les connaissances de manière à générer un processus d'innovation continue et une véritable gestion des connaissances. L'exemple de Singapore Airlines, un des acteurs dominants dans l'industrie de l'aviation commerciale, offre quelques pistes intéressantes sur ce point. Cette compagnie qui allie avec succès réduction de coûts et excellence de service a mis en place un processus d'innovation « distribuée » entre chacun de ses départements : le programme «Idées du personnel en action » (Staff Ideas in Action) encourage un flot de nouvelles idées et se focalise sur l'amélioration continue (Heracleous, Wirtz, 2014 ; Heracleous, Wirtz, Johnston, 2004). Les idées des employés sont captées et une partie non négligeable est implantée par la suite, faisant l'objet d'un processus émergent. En parallèle, il existe également un processus d'innovation « centralisée » où les employés présentant les idées les plus innovatrices sont repérés et amenés à participer à un cercle "élite » regroupant quelques employés de tous les départements confondus, pour réfléchir à la stratégie globale de l'entreprise (Heracleous, Wirtz, 2014 ; Heracleous, Wirtz, Johnston, 2004). Des acteurs transversaux comme les directeurs de vol pourraient aider à mettre en valeur les contributions des agents, au-delà encore d'une rétroaction positive à la fin du vol, 
comme il est fréquent chez Cie 3. Ce pourrait être l'occasion de connecter les agents montrant un potentiel à un groupe d'innovation pour accroître ses capacités d'apprentissage (Wenger, 2010) et constituer une perspective d'évolution de carrière (Karatepe, Vatankhah, 2015). Par ailleurs, la possibilité d'une CoP pilotée constituée de directeurs de vol, fonctionnant en mode virtuel (Amin, Roberts, 2008), à distance, pourrait être étudiée (Tremblay, 2005 ; Cohendet et al., 2010).

\section{Engager les agents et valoriser leurs connaissances dans un contexte difficile}

Pour être en mesure de participer à de telles institutions, des incitatifs sont nécessaires, ou au moins une libération du temps de vol. Dans un contexte où l'on cherche à maximiser la force de travail et la flexibilité des agents à bord, ceci peut être difficile. Par ailleurs, les travailleurs ne souhaitent pas forcément faire de temps supplémentaire, un problème déjà rapporté par ailleurs. Les agents viennent avant tout au travail pour gagner leur vie (Chanal, 2000 ; Wenger, 1998), d'où la difficulté d'une gestion des connaissances dans ce type d'industrie. Cependant, ce constat ne devrait pas décourager d'en mettre une en place. Bon nombre de participants au sein de Cie 3 se sentaient « fiers » de travailler pour cette compagnie, motivant des comportements de citoyenneté d'apprentissage (Wenger, 2010) comme une générosité dans le partage de connaissances tacites ou une volonté d'améliorer les pratiques. Nos participants canadiens tâchaient plutôt de tirer une valorisation à travers le travail effectué en cabine seulement. L'engagement organisationnel - et non seulement professionnel - nous paraît constituer un ingrédient important pour une gestion des connaissances systémique à échelle organisationnelle. Cependant, une gestion par l'engagement peut être difficilement envisageable au sein de compagnies où les relations de travail sont historiquement tendues, comme dans bon nombre de compagnies aériennes établies (Bamber et al., 2009). Certaines ont essayé de remplacer une culture de contrôle par une culture de l'engagement, toutefois ont échoué pour de multiples raisons, entre autres, une identité éclatée suite à de multiples fusions, acquisitions et restructurations organisationnelles (Bamber et al., 2009). On ne change pas une culture organisationnelle du jour au lendemain. Ceci renforce notre argument principal selon lequel les compagnies aux relations de travail tendues (p. ex. Cie 1) ont intérêt à investir dans des acteurs transversaux satisfaisant les caractéristiques que nous avons identifiées, tout en respectant et en valorisant leur appartenance multiple (Wenger, 2010) et en leur donnant les ressources nécessaires pour accomplir leur rôle (Wenger, 2002). 
De manière plus générale, il paraît important de valoriser les connaissances des employés à l'heure où l'identité professionnelle des agents est menacée par la concurrence accrue au sein du transport aérien et les attentes changeantes et de plus en plus exigeantes des consommateurs. L'intelligence émotionnelle et le savoir-faire des agents de bord devraient être valorisés et ceux-ci devraient être récompensés pour leurs prouesses au travail dans un contexte de restriction de coûts croissante où, comme nous l'avons vu, certains parviennent à allier excellence de service et réduction de coûts (Heracleous, Wirtz, 2014 ; Nameghi, Ariffin, 2013).

\section{Conclusion}

Une écologie de la connaissance reposant sur la performance d'employés de service en contact avec la clientèle est-elle possible ? Notre recherche montre que les acteurs transversaux à la frontière des communautés professionnelles ont le potentiel de mener vers cet idéal. À travers la reconnaissance du rôle transversal des directeurs de vol, nous avons amené de nouvelles implications théoriques sur la notion de transversalité développée par Wenger (2010). D'une part, cet acteur traduit la logique de la direction dans la logique de la communauté professionnelle. D'autre part, il protège les façons de faire de la communauté, quitte à contourner les standards commerciaux. Il a la confiance du PNC comme du management, fait preuve de leadership participatif et d'un haut niveau de compétence. Cependant, un manque de canaux de communication ascendants semble bloquer la possibilité de partager les connaissances. Nous avons observé que la logique horizontale du PNC est peu reconnue par la direction. Ceci ne va pas sans causer certaines tensions identitaires au sein du PNC. Cette question mériterait d'être prise en compte, notamment en faisant des agents des travailleurs du savoir. Enfin, s'il demeure toujours une tension entre les axes d'imputabilité horizontal et vertical, il importe que ces deux structures demeurent en interaction (Wenger, 2010).

\section{Limites}

Comme toute étude, la nôtre comporte certaines limites. Il aurait été intéressant de comparer l'expérience du PNC à la vision des gestionnaires au sein de ces compagnies aériennes. Cependant, nous ne sommes pas parvenues à les rejoindre en nombre suffisant pour effectuer des entretiens. Une étude de cas comportant une analyse du point de vue des gestionnaires au sujet 
de l'innovation et de la gestion des connaissances permettrait de compléter notre recherche, et d'étudier plus précisément la possibilité d'une écologie de la connaissance. Ensuite, mentionnons que certaines différences culturelles entre les pays à l'étude pourraient aussi expliquer certaines différences dans les attitudes des participants (Hofstede, 2003), de sorte qu'il n'est pas certain que l'on puisse transférer les pratiques d'un pays à l'autre. Cependant, pour les cas de succès, il serait sans doute intéressant de tout au moins tenter les transferts de pratiques et de connaissances.

\section{RÉFÉRENCES}

ALJUWAIBER, A. (2016), Communities of Practice as an Initiative for Knowledge Sharing in Business Organisations: A Literature Review, Journal of Knowledge Management, 20(4), 731-748.

AMIN, A., ROBERTS, J. (2008), Knowing in Action: Beyond Communities of Practice, Research Policy, 37(2), 353-369.

BAMBER, G. J., GITTELL, J. H., KOCHAN, T. A., VON NORDENFLYCHT, A. (2009), Up in the Air: How Airlines Can Improve Performance by Engaging Their Employees, Ithaca, NY, ILR Press.

BARNEY, J.B. (1991), Firm Resources and Sustained Competitive Advantage, Journal of Management, 17, 99-120.

BARNIER, L. M. (1999), Personnel navigant commercial, d'un savoir intuitif à un savoir d'expertise, Formation Emploi, 67, 25-46.

BECHKY, B. A. (2003), Sharing Meaning across Occupational Communities: The Transformation of Understanding on a Production Floor, Organization Science, 14(3), 312-330.

BECKER, F. (2007), Organizational Ecology, Knowledge Networks, California Management Review, 49(2), 1-20.

BELOBABA, P. B., SWELBAR, W. S., ODONI, A. R. (2016), Critical Issues and Prospects for the Global Airline Industry, in Belobaba, P, Odoni, A., Barnhart, C. (eds), The Global Airline Industry, 2nd edition, John Wiley \& Sons, 487-500.

BOWEN, D. E., LAWLER, E. E. (1992), The Empowerment of Service Workers: What, Why, How and When, Sloan Management Review, 33(3), 1-31.

BROWN, J. S. (1999, 2002), Learning, Working $\mathcal{F}$ Playing in the Digital Age, URL: http:// serendip.brynmawr.edu/sci_edu/seelybrown/seelybrown.html

BROWN, J. S., DUGUID, P. (1991), Organizational Learning and Communities of Practice: Toward a Unified View of Working, Learning, and Innovation, Organization Science, 2(1), 40-57.

BROWN, J. S., DUGUID, P. (1998), Organizing Knowledge, California Management Review, 40(3), 90-111.

CHANAL, V. (2000), Communautés de pratique et management par projet, Management, $3(1), 1-30$. 
CHATTI, M. A. (2012), Knowledge Management: A Personal Knowledge Network Perspective, Journal of Knowledge Management, 16(5), 829-844.

COHENDET, P., ROBERTS, J., SIMON, L. (2010), Créer, implanter et gérer des communautés de pratique, Gestion, 35, 31-35.

DAVEL, E., TREMBLAY, D. G. (2011), Formation et apprentissage organisationnel : la vitalité de la pratique, Sainte-Foy, Presses de l'Université du Québec.

DUBEY, G., MARTIN, R. (2001), Les coulisses du ciel, Quaderni, 44, 89-100.

DRISKO, J. W., MACHI, T. (2016), Content Analysis, New York, Oxford University Press.

ECKERT, P. (1989), Jocks and Burnout: Social Categories and Identity in the High School, New York, Teachers College Press.

GLASER, B. G., STRAUSS, A. L. (1967), The Discovery of Grounded Theory, Hawthorne, N.Y., Aldine Press.

GJELSVIK, M. (2002), Hotels as Learning Arenas, Scandinavian Journal of Hospitality and Tourism, 2, 31-48.

GHERARDI, S. (2009), Communities of Practice or Practices of a Community?, in Armstrong, S., Fukami, C. (eds), The Sage Handbook of Management, Learning, Education and Development, Londres, Sage Publications, 514-530.

GUEUDAR-DELAHAYE, A. (2012), Faire équipe entre ciel et terre : le cas des navigants aériens, Nouvelle revue de psychologie, 14, 51-66.

HABHAB-RAVE, S. (2010), Le rôle des communautés de pratique dans le processus de gestion des connaissances des entreprises innovantes : une étude de cas par comparaisons intersites, Revue des Sciences de Gestion, 241, 43-54.

HALLIN, C. A., MARNBURG, E. (2007), Knowledge Management in the Hospitality Industry: A Review of Empirical Research, Tourism Management, 1-16, doi:10.1016/j. tourman.2007.02.019

HARVEY, J. F., COHENDET, P., SIMON, L., DUBOIS, L. E. (2013), Another Cog in the Machine: Designing Communities of Practice in Professional Bureaucracies, European Management Journal, 31, 27-40.

HERACLEOUS, L., WIRTZ, J., JOHNSTON, R. (2004), Cost-effective Service Excellence: Lessons from Singapore Airlines, Spring, 15(1), 33-38.

HERACLEOUS, L., WIRTZ, J. (2014), Singapore Airlines: Achieving Sustainable Advantage through Mastering Paradox, The Journal of Applied Behavioral Science, 50(2), 150-170.

HIPP, C., GRUPP, H. (2005), Innovation in the Service Sector: The Demand for Servicespecific Innovation Measurement Concepts and Typologies, Research Policy, 34, 517535.

HOFSTEDE, G. (2003), Culture's Consequences: International Differences in Work-Related Values, 2nd edition, Thousand Oaks, Sage Publications.

KAHLE, E. (2002), Implications of "New Economy" Traits for the Tourism Industry, Journal of Quality Assurance in Hospitality $\mathcal{E}$ Tourism, 3(3-4), 5-23.

KARATEPE, O. M., VATANKHAH, S. (2015), High-Performance Work Practice, Career Satisfaction, and Service Recovery Performance: A Study of Flight Attendants, Tourism Review, 70(1), 56-71. 
LAVE, J., WENGER, E. (1991), Situated Learning: Legitimate Peripheral Participation, Cambridge, Cambridge University Press.

LESSOR, R. (1984), Social Movements, the Occupational Arena and Changes in Career Consciousness, Journal of Occupational Behavior, 5(1), 37-51.

LINKVIST, L. (2005), Knowledge Communities and Knowledge Collectivities: A Typology of Knowledge Work in Groups, Journal of Management Studies, 42(6), 1189-1210.

MUTCH, A. (2003), Communities of Practice and Habitus: A Critique, Organization Studies, 24, 3, 383-401.

NAMEGHI, E. N. M., ARIFFIN, A. A. M. (2013), The Measurement Scale for Airline Hospitality: Cabin Crew's Performance Perspective, Journal of Air Transport Management, 30, 1-9.

NONAKA, I., KONNO, N. (1998), The Concept of "Ba": Building a Foundation for Knowledge Creation, California Management Review, 40(3), 40-54.

NONAKA, I., TAKEUCHI, H. (1995), The Knowledge-creating Company: How Japanese Companies Create the Dynamics of Innovation, New York, Oxford University Press.

ORR, J. E. (1990), Sharing Knowledge, Celebrating Identity: War Stories and Community Memory in a Service Culture, in Middleton, D. S., Edwards, D. (eds), Collective Remembering: Memory in Society, Beverly Hills, Sage.

PATA, K. (2009), Revising the Framework of Knowledge Ecologies: How Activity Patterns Define Learning Spaces?, in Lambropoulos, N., Romero, M. (eds), Educational Social Software for Context-Aware Learning: Collaborative Methods $\mathcal{B}$ Human Interaction, Hershey, NY, Information Science Reference, 241-267.

PATTON, M. Q. (1999), Enhancing the Quality and Credibility of Qualitative Analysis, Health Services Research, 34(5-2), 1189-1208.

POIROT-DELPECH., S. (2016), Le collectif éphémère des personnels navigants sur les long-courriers, Socio-anthropologie, 33, 127-141.

POLANYI, M. (1958), Personal Knowledge: Towards a Post Critical Epistemology, Chicago, IL, University of Chicago Press.

RETNA, K. S., NG, P. T. (2011), Communities of Practice: Dynamics and Success Factors, Leadership $\mathcal{E}$ Organization Development Journal, 31(1), 41-59.

ROBERTS, J. (2006), Limits to Communities of Practice, Journal of Management Studies, 43(3), 623-639.

SAVOIE-ZAJC, L. (1997), L'entrevue semi-dirigée, in Gauthier, B. (dir.), Recherche-sociale : de la problématique à la collecte des données, $3^{e}$ éd., Sainte-Foy, Presses de l'université du Québec.

SAINT-ONGE, H., WALLACE, D. (2003), Leveraging Communities of Practice for Strategic Advantage, St. Louis, Butterworth-Heinemann.

SIEMENS, G. (2006), Knowing Knowledge, Lulu.com.

SIMPSON, R. (2014), Gender, Space and Identity Male Cabin Crew and Service Work, Gender in Management: An International Journal, 29(5), 291-300.

STAR, S. L., GRIESEMER, J. R. (1989), Institutional Ecology, Translation and Boundary Objects: Amateurs and Professionals in Berkley's Museum of Vertebrate Zoology, Social Studies of Science, 19(3), 1907-1939. 
TREMBLAY, D. G. (2015), Emploi et gestion des ressources humaines dans l'économie du savoir, Sainte-Foy, Presses de l'Université du Québec.

TREMBLAY, D. G. (2014), L'innovation technologique, organisationnelle et sociale, Québec, Presses de l'université du Québec.

TREMBLAY, D. G. (2005), Les communautés de pratique : quels sont les facteurs de succès ?, Revue internationale sur le travail et la société, 3(2), 52-79.

WENGER, E. (1998), Communities of Practice: Learning, Meaning and Identity, New York, Cambridge University Press.

WENGER, E. (2000), Communities of Practice and Social Learning Systems, Organization, 7, 225-246.

WENGER, E. (2009), Learning Capability in Social Systems, EQUAL Final Report.

WENGER, E. (2010), Communities of Practice and Social Learning Systems: The Career of a Concept, in Blackmore, C. (ed.), Social Learning Systems and Communities of Practice, Springer, London, 179-198.

WENGER, E., MCDERMOTT, R., SNYDER, W. M. (2002), Cultivating Communities of Practice: A Guide to Managing Knowledge, Boston, Harvard Business School Press.

WHITELEGG, D. (2007), Working the Skies: The Fast-Paced, Disorienting World of the Flight Attendant, New York University Press. 